\title{
SMGP Schweizerische Medizinische Gesellschaft für
}

\section{Phytotherapie}

Kurs-Sekretariat (8-10 Uhr) Keltenstrasse 40 CH-8044 Zurich (Schweiz) Fax/Tel. (00 41) 01/2 521879

ESCOP Scientific Committee tagte

in Baden $(\mathrm{CH})$

ESCOP-was ist das? Warum ESCOP?

M. Frater-Schröder,

B. Meier, M. Mennet-von Eiff

Im Juni 1989 schloss sich eine kleine Gruppe motivierter Phytotherapie-Spezialisten, Vertreter ihrer nationalen Gesellschaften, zu einer neuen Gesellschaft (ESCOP) zusammen, welche sich das Ziel setzte, den wissenschaftlichen Status der Phytotherapeutika zu fördern und harmonisierend in der Gesetzgebung für diese Mittel in Europa zu wirken.

Was ist ESCOP?

ESCOP - diese Abkürzung bedeutet «European Scientific Cooperative on Phytotherapy» unterstützt und fördert die wissenschaftliche Tätigkeit im Bereich der Phytotherapie. Sie koordiniert die Bemühungen zur Harmonisierung der Anforderungen an Phytopharmaka in den verschiedenen europäischen Ländern.

ESCOP wurde am 18. Juni 1989 in Köln von Delegierten der phytotherapeutischen

Gesellschaften aus sechs Nationen gegründet: Belgien. Schweiz, Deutschland, Frankreich, Niederlande und Grossbritannien. Seither sind Italien. Irland, Griechenland und Schweden dazugekommen. 12 weitere nationale Gesellschaften bewerben sich um die Mitgliedschaft. Sie sind derzeit im Status von «Associated Members*.

Mitglieder der SMGP wirkten von Anfang an in den verschiedenen ESCOP-Kommissionen mit und garantieren eine Vertretung und ein Mitspracherecht bei Beratungen über Arznei-pflanzen in einem Europäischen Gremium.

Warum ESCOP?

Die konkreten Ziele der ESCOP sind:

Koordination der wissenschaftlichen Tätigkeit zur Unterstützung der Phytopharmaka

Förderung der Akzeptanz von Phytopharmaka, speziell bei allgemein praktizierenden Ärzt-Innen und in der Selbstmedikation

Unterstützung der klinischen und experimen-tellen Forschung auf dem Gebiet der Phytotherapie

-Förderung der Zusammenarbeit zwischen nationalen Phytotherapie-Gesellschaften und

Europäischen Behörden. Harmonisierung der unterschiedlichen nationalen Anforderungen an

Phytopharmaka - Aufarbeitung und Erweiterung der international vorhandenen

wissenschaftlichen Erkennt-nisse und des praktischen Wissens im Gebiet der Phytotherapie.

Was sind Phytopharmaka? 
Gemäss ESCOP und in Übereinstimmung mit den Europäischen Kriterien in «The Note for Guidance: Quality of Herbal Remedies» und in «Council Directive 65/65 EEC» können Phytopharmaka folgendermassen definiert werden:

Phytopharmaka (pflanzäche Heilmittel) sind ArzПeimittel, deren wirksame Komponenten aus Pflanzen, Pflanzenteilen oder deren Kombina-tionen, entweder unverarbeitet oder verarbei-tet, bestehen.

Wie werden die Ziele von ESCOP realisiert?

Die Ziele der ESCOP werden vorwiegend mit Hilfe des wissenschaftlichen Ausschusses, des «Scientific Committee», angesteuert. Dieses Komitee ist seit mehr als 5 Jahren tätig und besteht aus jeweils 1 bis 2 Delegierten aus den erwähnten Europäischen Ländern. Die Delegierten sind ausnahmslos Expertlnnen (Ärztln-nen, Pharmazeutlnnen, Chemikerlnnen und Botanikerlnnen) auf dem Gebiet der Phytopharmaka. Es werden Monographien über Arzneipflanzen und ihre therapeutischen An-wendungen erstellt. Sie werden mit moderner. aktueller und ergänzend auch mit älterer, an-erkannter Fachliteratur dokumentiert. Diese wissenschaftlichen Zusammenfassungen oder Monographien werden unter dem Namen SPC (Summary of Product Characteristics) bei den Europäischen Behörden eingereicht. Die SPCs sollen zur

Harmonisierung von Registrierungs-Vorschriften innerhalb der Europäischen Union beitragen. Den Stellenwert dieser wissenschaftlichen Tätigkeit hat die Europäische Union vor einem Jahr mit einem Beitrag aus dem Forschungs-fonds BIOMED (BIOMEDical and Health Research Programme of the European Community) bestätigt. Dieses 3 Jahre laufende Forschungsprogramm umfasst die Monogra-phierung von 46 Arzneipflanzen. Das BIOMEDForschungsprogramm beinhaltet zudem die Entwicklung von Methoden zum Nachweis von Wirksamkeit und Unbedenklichkeit der Phytopharmaka. Innerhalb dieses Rahmens soil der komplexen Natur der Phytopharmaka voll Rechnung getragen werden. Ausserdem soil in einer Pilotstudie ein «Pharmacovigilance System* erarbeitet werden, welches Meldungen über unerwünschte Ereignisse in Zusammen-hang mit der Anwendung von Phytopharmaka europaweit dokumentiert und beurteilt.

Die Bemühungen der ESCOP wurden mit einer ersten offiziellen Anerkennung von 4 Monographien als SPCs durch das CPMP (Committee for Proprietary Medicinal Products) der Europäischen Gemeinschaft hono-riert. Es handelt sich hierbei um die SPCs von Frangulae cortex, Sennae folium, Sennae fructus acutifoliae und Sennae fructus angu-stifoliae.

Prof. Dr. B. Meier, Präsident der SMGP, eröffnete die letzte Sitzung, die am 20. Januar in Baden $(\mathrm{CH})$ stattfand, mit einem Grusswort. Er bestätigte die Delegierten in ihren Bemühungen, Pflanzen-SPCs zu erstellen, und dankte für ihr wertvolles Engagement. Die phytotherapeutischen Wirkprinzipien sind primär den Pflanzen und nicht den daraus gewonnenen Produkten zuzuordnen. Deshalb ist die Arbeit an Pflanzen-SPCs richtig und wichtig. Es obliegt den Herstellern, ihre Produkte so zu entwickeln und herzustellen, dass sie den durch die SPCs festgelegten Anwendungsbereichen gerecht werden. Der neue Trend, nur noch sehr eng definierte Extrakte zu monographieren, ist aus der Sicht der SMGP abzulehnen, da er im Prinzip jede Innovation behindert und einen Status quo zementiert.

Sind Sie an weiteren Informationen über

ESCOP interessiert?

Möchten Sie am nächsten ESCOP-Sympo-sium teilnehmen? Möchten Sie das «European Phytotelegram official Newsletter of ESCOP» erhalten?

Schreiben Sie bitte an: 
ESCOP Secretariat Uiterwaardenstraat 13 NL-8081 HJ Elburg (The Netherlands)

(C) 1995 S. Karge $\mathrm{GmbH}$, Freiburg

Aufnahmeantrag

Hiermit stelle ich den Antrag, in die SMGP Schweizerische Medizini- Universitäre

sche Gesellschaft für Phytotherapie als ordentliches Mitglied aufgenommen Ausbildung

zu werden.

Personalien

Name

Titel

Vorname

Geb.-Datum

Stellung Spital o

andere

Praxis LJ Apotheke LJ

Adresse

Strasse. PLZ_

Tel.

Ort.

/

Ort/Datum

Unterschrift

Wort des Präsidenten

Liebe Kolleginnen und Kollegen,

In der Politik ist es üblich, nach hundert Tagen Bilanz zu ziehen. Unser Verein, die SMGP, ist kleiner, deshalb darf die erste Bilanz etwas später sein. In den fünf Monaten, seit ich das Amt übernommen habe, konnten sich die Vorstandsmitglieder über zuwenig Arbeit nicht beklagen. Die SMGP wird zum An-sprechpartner.

Anfragen zu den Möglichkeiten der Phytotherapie bei Krebserkrankungen (sie sind - von der komplexen Misteltherapie abgesehen - ad-iuvanter Art), die Vernehmlassung zur Verord-nung zum neuen Krankenversicherungsgesetz, das Interesse von Krankenkassen an Phytotherapie und an unserer Ausbildung beschäftigten uns. Daneben wurde zum dritten Durchgang unserer Ausbildung gestartet. Dies mit erfreu-lichem Erfolg, sind doch die Kurse weiterhin gut ausgebucht. Eine junge Generation neuer Phytotherapeutinnen und Phytotherapeuten konnte gewonnen werden. Dementsprechend positiv verläuft die Entwicklung unserer Mit-gliederzahl wenn es so weiter geht wie zuletzt, erreichen wir vielleicht noch dieses Jahr die Zahl von zweihundert. Als bisherige Mitglieder können Sie ganz besonders dazu beitragen. dieses Ziel zu erreichen - wenn Sie Ihre Kolle-

ginnen und Kollegen auf die SMGP aufmerk-sam machen. Wir selbst wollen die PR-Aktivitä-ten intensivieren - die in etlichen Zeitschriften erfolgte Berichterstattung von unserer letzten Tagung war ein erster Schritt dazu.

Zahlreiche Aufgaben stehen vor uns: Die 10. Schweizerische Tagung für Phytotherapie soil einen Ausblick in die Zukunft bringen und verspricht spannende Informationen zum Stand der Dinge bei den Krankenkassen, bei der IKS, in der Forschung und im Spital mit namhaften 
Referentinnen und Referenten. Der Termin (26. Oktober) sollte unbedingt in jeder Agenda vermerkt bleiben. Im Hinblick auf die nächste Generalversammlung am gleichen Tag oder auf jene von 1996 müssen die Statuten revi-diert werden, und ein neues Planungspapier 1996-2000 muss das bisherige ablösen. Ferner möchten wir am Ball bleiben, wenn es darum geht, unsere Ausbildung (die Herr Professor Sailer vom Universitätsspital Zurich sehr beein-druckt hat) als offïzielle Weiterbildung für die Ärzte- und Apothekerschaft anzuerkennen und phytotherapeutische Behandlungen weiterhin oder neu durch die Grundversicherung der Krankenkassen decken zu lassen. Diesbezüglich sind wir für jede Unterstützung dankbar. Ostern 1995

Prof. Dr. Beat Meier, Präsident SMGP

Neue Mitglieder

(März/April 1995)

Frau E. Bischof-Mark, eidg. dipl. Apothekerin ETH, Lüsisstrasse 4B, CH-8880 Walenstadt Herr Dr. med. L. Kober, Innere Medizin FMH, Domstrasse 3, CH-4144 Arlesheim Frau Dr. sc. nat. Anne Rehwald, Zentralstrasse 144, CH-5430 Wettingen Herr Dr. med. R.N. Lietha, Zürcherstrasse 146, CH-8640 Rapperswil Frau Chantal D. König, eidg. dipl. Apothekerin,

Kaltbrunnenstrasse 7, CH-4054 Basel Herr U. Graf, eidg. dipl. Apotheker,

Mittlere Gasse 1, CH-8590 Romanshorn

176

Gesellschaftsmitteilungen · Society Bulletins 